\title{
THE AURICULOTEMPORAL SYNDROMEE A CLINICAL AND PHARMACOLOGIC STUDY
}

\author{
By A. S. FREEDBERG, ROBERT S. SHAW and M. J. McMANUS \\ (From the Department of Medicine, Harvard Medical School, and the Medical Service and \\ Research Laboratories, Beth Israel Hospital, Boston)
}

(Received for publication May 26, 1948)

The auriculotemporal syndrome which develops after injury to the parotid gland, consists of flushing and profuse sweating, upon eating, over the cutaneous distribution of the auriculotemporal nerve on the injured side. Although there have been few cases reported since the original description by Frey (1) in 1923, the syndrome is not rare, having been noted repeatedly, for example, in clinics observing patients after operation on a parotid gland $(2,3)$. The syndrome is of interest to the physiologist because it affords an opportunity to study the consequences of denervation of cholinergic end organs. It is our purpose to present three instances of this syndrome with some observations relating to its mechanism.

\section{CASE HISTORIES}

Case 1: J. B., a 68 year old man, BIH No. 78777, entered the hospital on $9 / 29 / 44$ complaining of transient weakness and dysarthria. These symptoms were believed to be consequent to a recent cerebrovascular accident.

Forty-one years ago, while in the Russian army, the patient had had typhoid fever, complicated by right parotitis. Two attempts to drain the parotid gland by vertical incisions were unsuccessful. Two incisions, $2 \mathrm{~cm}$. lateral to the right upper border of the thyroid gland, resulted in drainage for approximately 50 days. Approximately one month after the successful drainage, and two months after the onset of the parotitis, the patient noticed the development of profuse sweating over the right side of the face while eating. Although ingestion of many foods was followed by sweating, the phenomenon was particularly marked when apples were eaten. On each occasion just prior to the onset of sweating the patient experienced a sensation of warmth over the involved area. Redness, swelling and pain, however, were absent; at no time was numbness noted over the involved area. The amount of sweating had become less pronounced during the previous three years.

On physical examination, scars on the face and neck were noted. The opening of the right Stenson's duct was protuberant; saliva could not be expressed from it. The orifice of the left duct appeared normal and saliva was easily expressed. Perception of heat and cold was normal and equal on both sides of the face. There was cutaneous hypaesthesia in the distribution of the right auriculo- temporal nerve. There was a right facial weakness and ptosis of the right upper eyelid; the latter was said to have been present since the patient's recent cerebral vascular accident. The cranial nerves were otherwise normal.

Case 2: T. H., a 63 year old man, MGH No. 484270 , with a history of recent sinusitis, had had bilateral suppurative parotitis, of unknown etiology, twenty-five years previously; this had been drained through incisions over the glands on both sides. Several weeks after operation the patient noted the occurrence of profuse sweating and a feeling of warmth on the left side of his face when eating. The phenomenon had continued until admission. It was brought on by eating any food, especially apples. At no time had the patient noticed any numbness or paralysis of his face. Physical examination was not remarkable except for the old drainage scars over both parotid glands. The parotid glands were normal to palpation and saliva was easily expressed from both ducts. Sensation over the face was normal and the function of other cranial nerves was apparently intact.

Case 3: D. E. S., a 49 year old married woman (referred by Dr. I. T. Nathanson), had had a tumor of the right parotid gland removed in 1942. Approximately oni year later she noted the onset of sweating following the eating of various foods. The sweating involved the right side of the face in the region of the incision. Rarely a sense of heat was noted but visible flushing was never observed. No specific food was noted to be particularly effective in producing the sweating reaction. Although on rare occasions a whole meal did not produce sweating, hot tea by itself occasionally produced profuse perspiration. She had had mumps as a child, but with no sequelae.

\section{OBSERVATIONS}

Observations in Case 1: The observations on sweating were made by means of the Minor starch-iodine test (4) which consists of applying a solution of iodine (15 cc. 1 per cent iodine, $5 \mathrm{cc}$. of castor oil and $80 \mathrm{cc} .95$ per cent alcohol) and after drying, lightly dusting the skin with starch and noting the onset of sweating which is signalized as black spots. Ten seconds after taking a bite of apple and while chewing, the patient noticed a feeling of warmth over the right face, and a slight flush was noted on this side. Five seconds later, sweating appeared in part of the area in- 


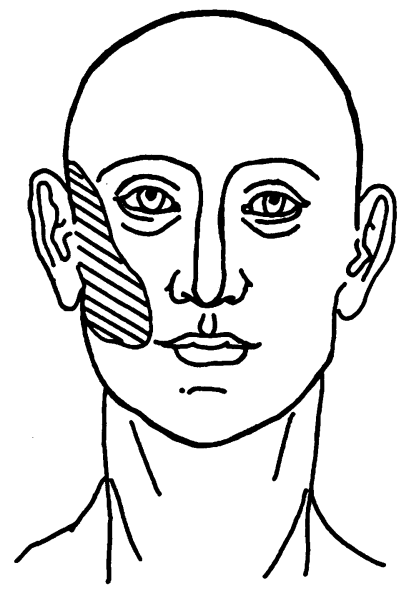

Fig. 1. The Distribution of Sweating Observed in Case 1, Represented by the Cross-Hatched Area

cluded in the distribution of the auriculotemporal nerve (Figure 1). The sweating could be induced by chewing any food, but was absent or slight when the patient chewed paraffin. The application of ice to the right face before chewing food resulted in a decreased amount of sweating in the chilled area. Sweating was absent when the patient held but did not chew food placed in his mouth. When the tongue was swabbed with vinegar, a small amount of sweating appeared. The response was independent of the region of the tongue swabbed. When the patient chewed a pledget of cotton soaked in vinegar, the amount of sweating, as judged by the extent and depth of the starch-iodine reaction, was more marked. Psychic salivation and sweating could not be induced. Sweating induced by the general application of heat to the body was irregularly diminished over the right side of the face.

Procainization of the right auriculotemporal nerve resulted in anesthesia over part of the distribution of the nerve. In the area of anesthesia sweating failed to appear after the usual stimulation. Novocaine injection ${ }^{1}$ of the right superior cervical ganglion resulted in a Horner's syndrome, suffusion of the conjunctiva, increased warmth, and dilation of the right hand veins. While the cervical sympathetics were blocked, eating an apple induced sweating in 45 seconds and 20 seconds in two successive trials. The patient was apprehen-

1 The authors are indebted to Dr. Reginald Smithwick for the procaine blocks in Cases 1 and 2 . sive during the whole period of injection and for a short time afterward.

A series of experiments was performed to determine whether there was hypersensitivity of the sweating mechanism in the involved area. In normal controls, local sweating was observed over and around intradermal wheals made by the injection of $0.1 \mathrm{cc}$. of various concentrations of acetylcholine bromide dissolved in isotonic saline solution. By using increasing dilutions, a measure of the sensitivity of the local sweating apparatus to acetylcholine might be obtained. While sensitivity was found to vary in different individuals and in different regions of the body, the end points were consistent. In this patient, intradermal wheals in the involved area on the right side of the face produced with $0.1 \mathrm{cc}$. of isotonic saline solution containing $0.0001-0.1$ gamma of acetylcholine bromide resulted in local sweating. Similar concentrations of acetylcholine in symmetrical positions on the left face of the patient did not produce sweating. A wheal on the left side of the face which contained 0.5 gamma acetylcholine per $0.1 \mathrm{cc}$. produced sweating.

Iontophoresis (3.4 milliamperes for ten minutes through a 1 sq. cm. pad) of a $1: 12,500$ solution of acetylcholine bromide in isotonic saline solution, produced sweating on the involved right side of the face and no sweating on the uninvolved left side on two attempts, with no sweating on either side on the third attempt. Ten mgm. of acetylcholine bromide injected subcutaneously did not result in facial sweating.

The possibility was examined that disturbances in vasomotor nervous function are present in the auriculotemporal syndrome. The variation in skin temperature of the involved areas in response to cooling and heating the body and extremities was measured and compared to symmetrical uninvolved areas. To determine the time of occurrence of the maximal response to heating and cooling, measurements of the skin temperature of a finger tip were made. The measurements of skin temperature were made at frequent intervals with a thermocouple. The experiment was performed in a constant temperature room, $20^{\circ} \mathrm{C}$. The patient was seated and the legs, arms and chest were uncovered. Within 20 minutes a distinct fall in finger tip temperature was observed (Figure 2) which reached a maximum in 70 minutes. The 
right ear skin temperature fell from $29.5^{\circ} \mathrm{C}$ to $25.5^{\circ} \mathrm{C}$ in this interval of time. At the arrow (70 minutes) one leg and one arm were immersed in running warm water ( $\mathrm{T} 42^{\circ}$ ); the patient was covered with blankets and hot water bottles were applied to the chest and back. Approximately 15 minutes later, a distinct and sustained rise in finger and left ear temperature occurred. The involved right ear, however, showed no rise in temperature in response to heating (Figure 2); furthermore, the increase in right facial skin temperature in response to heat was less than that observed in a symmetrical point on the uninvolved left side.

An attempt was made to measure and compare the secretion from the parotid glands in this patient. No saliva could be collected from the right parotid duct. Secretion from the left duct appeared normal.

Observations in Case 2: In this subject flushing was observed over the left side of the face ten seconds after he began to eat an apple, and in 15 seconds sweating was observed over the entire distribution of the auriculotemporal nerve on the left. A small area on the right cheek was also ob- served to sweat, although this had never been noted by the patient. Psychic salivation was easily induced, but the syndrome of flushing and sweating did not occur.

Sweating, distributed usually over the upper lip and nares, may be induced in normal subjects by the application to the tongue and chewing of red pepper or ginger. In this patient these foods resulted in sweating on the upper lip but did not induce sweating in the distribution of the auriculotemporal nerve.

Novocaine injection of the superior cervical sympathetic ganglion on the left resulted in a Horner's syndrome and an absence of sweating in response to heat. In the presence of this block, the auriculotemporal syndrome was observed as before, after the usual stimulus.

Intradermal wheals produced with .05 gamma acetylcholine bromide contained in $0.1 \mathrm{cc}$. isotonic saline solution produced sweating in the involved area on the left face, but none in the corresponding location on the right.

Saliva was collected from the right and left parotid ducts by means of silver cups attached by suction to the parotid gland duct orifices. No

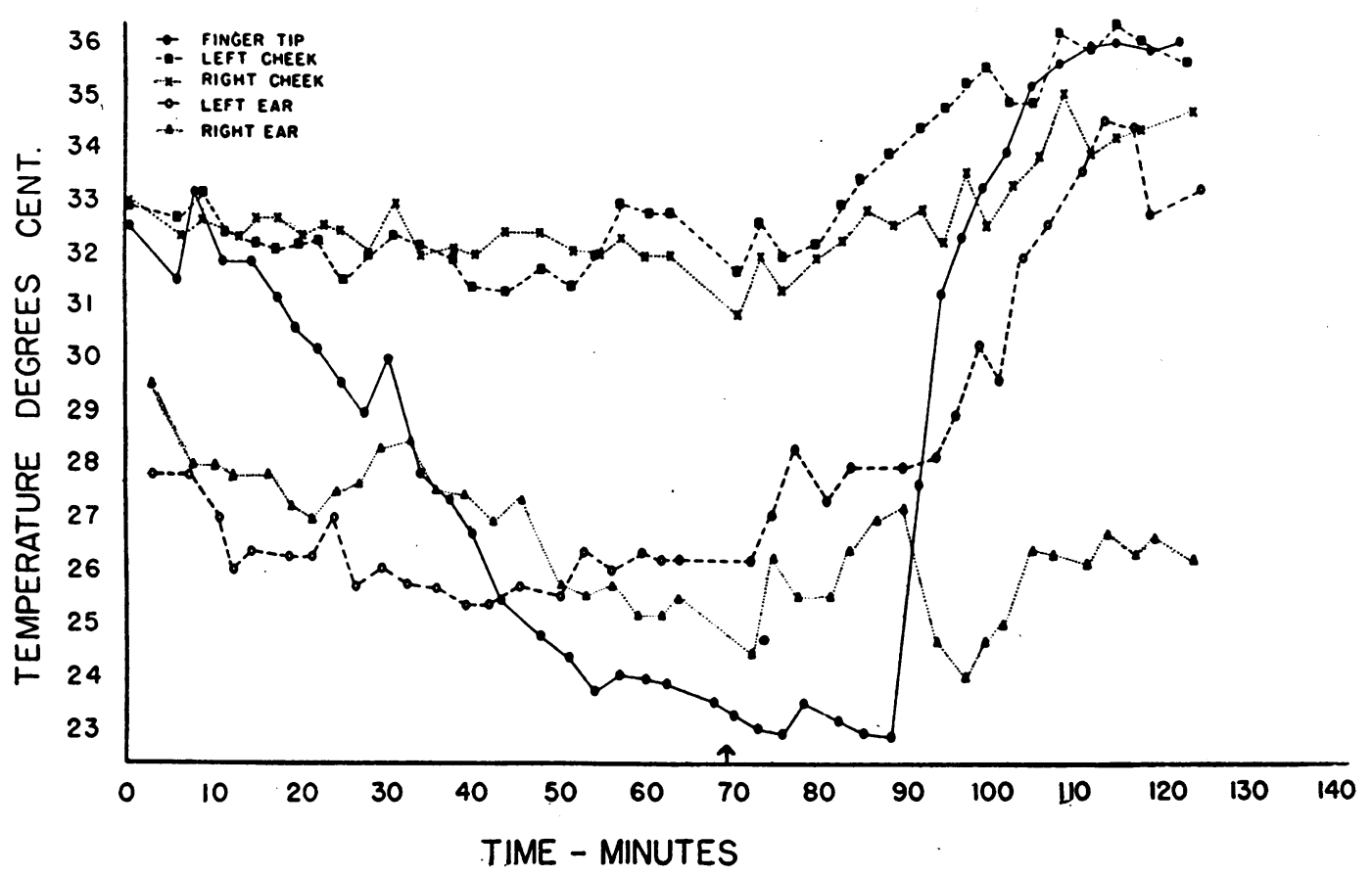

Fig. 2. Variations in Skin Temperature on Cooling and Heating the Body See text for description of figure. 


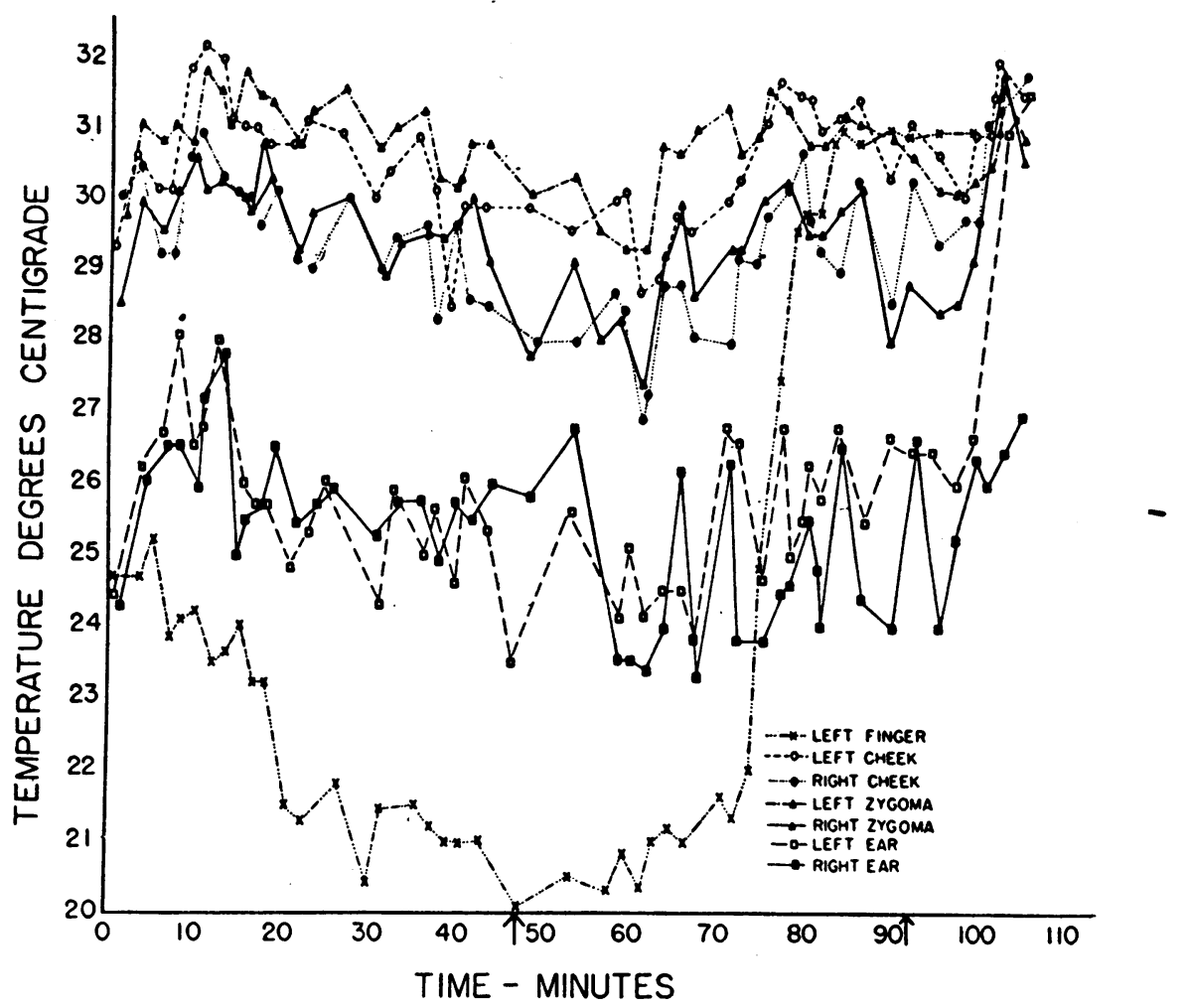

Fig. 3. Variation in Skin Temperature on Cooling and Heating the Body; Variations of the Skin Temperature Consequent to Eating in the Involved Skin Area in the Auriculotemporal Syndrome

See text for description of figure.

significant differences were found in the two samples in the amount or relative viscosity as measured by high pressure Ostwald viscosimeter.

Studies in the manner described in Case 1 were made of the variations of facial and ear skin temperature (Figure 3 ) with cooling and warming of the body and extremities. In response to cooling of the extremities and body a distinct fall in finger temperature was obtained. The changes in skin temperature of the ears and face were slight and no significant difference was observed between the right and left sides. At the first arrow (48 minutes) heat was applied and 25 minutes later a distinct rise in finger temperature occurred from approximately $21^{\circ} \mathrm{C}$ to $31^{\circ} \mathrm{C}$. After maximal finger vasodilation in response to heat had been obtained, eating an apple (arrow at 91 minutes) produced a definite increase in temperature not only on the involved left side of the face and ear but also on the right side. No asymmetry of facial sweating in response to heat could be observed.
Observations in Case 3: After the patient chewed an apple and on another occasion a lemon, sweating appeared in the region of the scar 16 and 12 seconds later, respectively. Chewing paraffin induced sweating in the same region in 16 seconds. Chewing ginger resulted in sweating in the region of the scar in 14 seconds and the upper lip in 30 seconds. Intradermal wheals were made in the involved area and in a symmetrical point on the left side of the face. On the right side sweating occurred over and around wheals containing .005 gamma acetylcholine bromide in $0.1 \mathrm{cc}$. isotonic salt solution. On the left side sweating was absent with wheals of a similar concentration and also with a concentration of 0.05 gamma in $0.1 \mathrm{cc}$. of isotonic salt solution.

\section{COMMENT}

There have been at least 35 instances of the auriculotemporal syndrome reported in the literature $(1,5-15)$. In the majority of cases the original episode involved both infection in and opera- 
tion on the parotid gland. The most common inciting circumstance was a parotitis, complicating typhus or typhoid fever, which had been drained surgically. The syndrome has also followed parotitis complicating syringomyelia, mumps, operation on the parotid without infection $(2,16)$, and parotitis of unknown etiology in the absence of suppuration and operation. The sweating makes its appearance from a few days (14) to three years (9) after the original episode, and lasts the rest of the patient's life. One case is reported with gradual disappearance of the syndrome over a three-year period (11). Uniformly the sweating appears over part or all of the distribution of the auriculotemporal nerve on the injured side. It is usually accompanied by visible flushing, and patients without visible flushing describe a sensation of warmth over the involved area. Minor disturbances in sensation and decreased sweating response to heat over the distribution of the auriculotemporal nerve of the involved side are frequently noted.

The severity of sweating varies from patient to patient, being slight in some (Case 3 ) and profuse in others (Cases 1 and 2). The studies described above suggest that relief may be obtained in instances of severe flushing and sweating by interruption of the efferent arc by alcohol injection or surgical section of the auriculotemporal nerve.

\section{Mechanism of auriculotemporal syndrome- anatomic pathway}

It appears that the disorder in the auriculotemporal syndrome lies in the efferent arc. The pathway of the afferent arc is variable since sweating may be initiated by stimulation of the seventh or ninth nerves and has been reported to result from psychic stimulation. The efferent pathway involves fibers in the region of the auriculotemporal nerve as demonstrated by the effect of procainization (Case 1). Since procainization of the superior cervical ganglion resulted in the disappearance of sweating in response to heat over the face but did not affect the occurrence of the auriculotemporal syndrome, it is clear that the fibers in the efferent arc do not pass through the cervical sympathetics.

\section{Proposed theories:}

I. It has been suggested (17) that the sweating phenomenon results from the diffusion of acetylcholine or cholinergic substances from the injured parotid gland. The speed of the reaction, the distribution in the course of the entire auriculotemporal nerve and the occurrence of the phenomenon in patients in whom the involved parotid gland is in all likelihood completely destroyed (Case 1) are against this interpretation.

II. Needles (12) noted the appearance of the auriculotemporal syndrome coincident with the closure of a salivary fistula, and felt that the syndrome might be caused by the irritation of sudomotor nerve fibers passing through or near the parotid gland by the swelling of the gland on mastication. This appears unlikely, since the syndrome may appear in the presence of an atrophied and functionless parotid gland (Case 1).

III. Ford has suggested (8) that the auriculotemporal syndrome may be explained by injury to the auriculotemporal nerve and subsequent aberrant regeneration of parotid secretory fibers along sudomotor and vasomotor pathways. The auriculotemporal nerve contains, in addition to sensory fibers, secretory fibers to the parotid gland, sudomotor fibers, and vasodilator fibers to the area of sensory distribution of the nerve (18); the parotid secretory fibers, sudomotor and vasodilator fibers are all cholinergic. The following facts are in support of this hypothesis :

1. The time interval between the original injury and the appearance of the syndrome, usually one month or longer, is consistent with a period of nerve degeneration and subsequent regeneration. One case, however, is reported (14) where the syndrome appeared two to three days following injury. 2. The frequently noted abnormalities in sensation and decreased sweating response to heat over the distribution of the auriculotemporal nerve, and, as in one of our cases, defective vasomotor response over the distribution of the auriculotemporal nerve, are consistent with injury to all components of the auriculotemporal nerve. Although hypersensitivity of the sweat glands over the affected region is consistent with the interpretation that the involved sweat glands have been partially denervated, it is difficult to explain the 
evident hypersensitivity after regeneration has taken place.

The resemblance of the case described by Uprus, Gaylor and Carmichael (16) to those exhibiting the auriculotemporal syndrome is so striking as to suggest a common mechanism. They described an instance of flushing and gustatory sweating over the distribution of the right cutaneous colli nerve appearing one to two years following the removal of cervical lymph glands through a horizontal incision over the mid right sternocleidomastoid muscle. In the involved area, sensations to pin prick and light touch were diminished. There was diminished sweating response to heat and an impaired vasomotor response after thermal stimuli. Procainization of the cutaneous colli nerve (sensory and vasomotor to the area) had no effect upon the flushing and the sweating reaction induced by food. Blocking the lingual nerve proximal to the origin of the chorda tympani resulted in cessation of salivation and the disappearance of the phenomena. In this instance the primary injury was to the cutaneous colli nerve and injury to the secretory fibres in the lingual nerve appears unlikely because of the level of the incision. With absence of injury to submaxillary secretory fibers, aberrant regeneration is unlikely in this case.

IV. The explanation offered here is that the auriculotemporal syndrome may represent the response of denervated and hypersensitive sweat glands to previously unapparent cranial sudomotor impulses. List and Peet observed (18) marked facial gustatory sweating following postganglionic cervical sympathectomy. In these patients, the sweating appeared following the eating of foods which were similar to those foods causing sweating in patients with the auriculotemporal syndrome. List and Peet (18) suggested that this type of sweating was an "exaggeration of normal gustatory sweating" caused by denervation hypersensitivity of the sweat glands, but they felt that denervation hypersensitivity was not the explanation of the auriculotemporal syndrome, because the diminution of heat sweating over the area involved in the auriculotemporal syndrome was so slight as to suggest that the denervation of the sweat glands was negligible. However, in the reported cases of the auriculotemporal syndrome, hypersensitivity to pilocarpine as manifested by early sweating in the involved area, and our observations of local hypersensitivity to acetylcholine in the involved regions as demonstrated by intracutaneous wheals or iontophoresis of acetylcholine, clearly show that there is a hypersensitivity of the sweat glands in the involved region. That hypersensitivity to cholinergic substances may occur in organs following denervation has been demonstrated in the submaxillary gland (19), skeletal muscle (20) and iris (21). In these latter studies hypersensitivity developed in from one to two weeks. Observations on the time relationship of the development of hypersensitivity following denervation of sweat glands are necessary.

Other data are available consistent with the interpretation that a cranial supply of sudomotor fibers exists. It is a common observation that facial sweating occurs in normal individuals with the eating of hot spicy foods. More unusually, it may occur with the eating of chocolate (9) and the drinking of vinegar (22). Gustatory sweating in normal individuals, as produced by the eating of ginger, has been studied by us. The sweating occurs maximally about the mouth and nose. Observations were also made in a patient who had a unilateral preganglionic cervical sympathectomy for Raynaud's syndrome. In this patient who had no Horner syndrome, there was absence of heat sweating on the denervated side. Gustatory sweating induced by eating ginger was marked on the normal side of the face but scarcely perceptible on the denervated side. This would suggest that the nervous pathways involved in gustatory sweating consequent upon the eating of ginger passed through the cervical sympathetics. The pathways concerned in the auriculotemporal syndrome do not, as demonstrated by the fact that the syndrome is not affected by cervical sympathetic block. Furthermore in Case 2 sweating induced by ginger was observed to occur independently from the auriculotemporal syndrome sweating. The failure to block the syndrome by procainization of the superior cervical ganglion (Cases 1 and 2), although heat sweating was abolished, is also consistent with the presence of cranial sudomotor fibers. That the facial sweat glands may have a 
dual innervation from the brain and cervical sympathetics was suggested by Takino (23) who observed both medullated and non-medullated nerves around the sweat glands of the face. The latter evidence is not decisive and further studies on this point are necessary.

It would seem, therefore, that the auriculotemporal syndrome, post-sympathectomy gustatory sweating and the case of Uprus, Gaylor and Carmichael represent sweating responses to nervous impulses in sweat glands made hypersensitive by denervation. Although as noted above, in the discussion of Ford's hypothesis, these nervous impulses may be from abnormally regenerated salivary secretory fibers, if cranial sudomotor fibers exist, they might be the efferent pathway in the three types of gustatory sweating under consideration. The three syndromes would then represent complete or partial severance of the sudomotor and vasomotor sympathetics to the involved regions leading to hypersensitivity of the sweat glands and exaggeration of normal and hitherto not obvious cranial sudomotor and vasomotor impulses.

\section{SUMMARY AND CONCLUSIONS}

1. The auriculotemporal syndrome is characterized by gustatory sweating and flushing over the cutaneous distribution of the auriculotemporal nerve. Three patients have been studied who exhibited this syndrome.

2. The gustatory sweating and flushing seen in the auriculotemporal syndrome is a manifestation of a reflex in which the efferent arc is through the auriculotemporal nerve or through adjacent autonomic fibers and not through the cervical sympathetic nerves. The afferent arc is nonspecific; the syndrome may be induced by stimulating the seventh and ninth nerves.

3. There may be a deficiency in sudomotor, thermo-regulatory and sensory innervation over the involved area.

4. A local hypersensitivity of the sweat glands in the involved region to acetylcholine bromide is present.

5. The mechanism of this syndrome is apparently related to denervation hypersensitivity of the sweat glands analogous to the hypersensitivity to adrenalin which occurs after post-ganglionic division of sympathetic fibers.

6. In instances of severe flushing and sweating, the described studies suggest that relief may be obtained by interruption of the efferent arc by alcohol injection or surgical section of the auriculotemporal nerve.

\section{BIBLIOGRAPHY}

1. Frey, L., Le Syndrome du Nerf Auriculo-temporal. Rev. neurol., 1923, 2, 97.

2. Nathanson, I. T., Personal Communication.

3. Bailey, H., Parotidectomy : Indications and Results. Brit. M. J., 1947, 1, 404.

4. Minor, V., Eines neues Verfahren zu der Klinischen Untersuchung der Schweissabsonderung. Deutsches Ztschr. f. Nervenh., 1928, 101, 302.

5. Kaminsky, S. D., Das "auriculo-temporale (Parotitis) Syndrom" bie Syringomyelie. Deutsches Ztschr. f. Nervenh., 1929, 109, 296.

6. Thomas, A., Le double reflex vaso-dilatateur et sudoral de la face consécutif aux blessures de la loge parotidienne; les pararéflexes. Rev. neurol., $1927,1,447$.

7. Fridberg, D., Das Auriculo-temporale Syndrom. Deutsches Ztschr. f. Nervenh., 1931, 121, 225.

8. Ford, F. R., Paroxysmal lacrimation during eating as a sequel of facial palsy (syndrome of crocodile tears) : report of four cases with a possible interpretation and comparison with the auriculotemporal syndrome. Arch. Neurol. \& Psychiat., 1933, 29, 1279.

9. Bassoe, P. N., Auriculotemporal syndrome and other vasomotor disturbances about the head: "auriculotemporal syndrome" complicating diseases of parotid gland, angioneurotic edema of the brain. M. Clin. of North America, 1932, 16, 405.

10. Noica and Bagdasar, Syndrome du nerf auriculotemporal. Rev. neurol., 1926, 1, 225.

11. Trioumphoff, A., Une forme particulière de l'hyperhidrose locale de la face. Presse méd., 1926, II, 86, 1350.

12. Needles, W., The auriculotemporal syndrome, with suggestion regarding therapy. Arch. Neurol. \& Psychiat., 1936, 35, 357.

13. Karnosh, L. J., The syndrome of the auriculotemporal nerve. Cleveland Clin. Quart., 1946, 13, 194.

14. Souques, A., Hyperhidrose unilatérale de la face consécutive à un traumatisme de la region sourcilière et provoquée par les excitations gustatives et par la chaleur. Des hémihyperhidroses d'origine cérébro-spinale. Rev. neurol., 1927, 2, 145.

15. Guttmann, L., and List, C. F., Zur Topik und Pathophysiologie der Schweissekretion. Ztschr. f. d. ges. Neurol. u. Psychiat., 1928, 116, 504.

16. Uprus, V., Gaylor, J. B., and Carmichael, E. A., Localized abnormal flushing and sweating on eating. Brain, 1934, 57, 443. 
17. List, C. F., and Peet, M. M., Sweat secretion in man. III. Clinical observation on sweating produced by pilocarpine and mecholyl. Arch. Neurol. \& Psychiat., 1938, 40, 269.

18. List, C. F., and Peet, M. M., Sweat secretion in man. IV. Sweat secretion of the face and its disturbances. Arch. Neurol. \& Psychiat., 1938, 40, 443.

19. Simeone, F. A., and Maes, J. P., Sensitization of the submaxillary gland by sympathetic denervation. Am. J. Physiol., 1939, 125, 674.
20. Cannon, W. B., Haimovici, H., Sensitization of motoneurones by partial "denervation." Am. J. of Phys., 1939, 126, 731.

21. Cannon, S., cited by Cannon, W. B., and Rosenblueth, A., Autonomic Neuro-Effector Systems. Macmillan Co., New York, 1937, p. 229.

22. Luchsinger, B., Die Schweissabsonderung und einige verwandte Secretionen bei thieren. Hermann Handbuch, phys. Leipzig, 1883, 1, 421.

23. Takino, cited by Kuno, Y., The Physiology of Human Perspiration. Churchill, Ltd., London, 1934, p. 9. 